\title{
Inhibition of HtrA2 alleviates inflammatory response and cell apoptosis in lipopolysaccharide-induced acute pneumonia in rats
}

\author{
XIN WANG \\ Department of Pediatrics, Shengjing Hospital of China Medical University, Shenyang, Liaoning 110022, P.R. China
}

Received January 6, 2020; Accepted May 18, 2020

DOI: $10.3892 / \mathrm{mmr} .2020 .11410$

\begin{abstract}
Pneumonia is one of the commonest causes of death worldwide. High-temperature requirement A2 (HtrA2) is a proapoptotic mitochondrial serine protease involved in caspase-dependent or caspase-independent cell apoptosis. UCF-101 (5-[5-(2-nitrophenyl) furfuryl iodine]-1,3-diphenyl-2-thiobarbituric acid), an inhibitor of $\mathrm{HtrA}$ 2, has a protective effect on organs in various diseases by inhibiting cell apoptosis. The aim of the present study was to explore whether UCF-101 has a protective effect on lungs in pneumonia. A lipopolysaccharide (LPS)-induced pneumonia model was established in rats. UCF-101 $(2 \mu \mathrm{mol} / \mathrm{kg})$ was used for treatment. Lung injury was detected by hematoxylin and eosin staining. Pro-inflammatory cytokines and oxidative stress-related factors were detected using corresponding test kits. TUNEL staining was used to measure the amount of cell apoptosis. Apoptosis-associated proteins were detected by western blot assay. The present study indicated pulmonary injury induced by LPS. Treatment with UCF-101 clearly alleviated this pulmonary damage and restored the levels of pro-inflammatory cytokines and oxidative stress-related factors. In addition, UCF-101 significantly reduced LPS-induced cell apoptosis, the release of HtrA2 and cytochrome from mitochondria to the cytoplasm and inhibited the expression of pro-apoptotic proteins. UCF-101 also restored the ATP level. The present results demonstrated that UCF-101 acts as a positive regulator of acute pneumonia by inhibiting inflammatory response, oxidative stress and mitochondrial apoptosis. The present study suggests UCF-101 as a potential candidate for pneumonia therapy.
\end{abstract}

Correspondence to: Dr Xin Wang, Department of Pediatrics, Shengjing Hospital of China Medical University, 9 Huaxiang Road, Tiexi, Shenyang, Liaoning 110022, P.R. China

E-mail: wangxx20@126.com

Key words: apoptosis, high-temperature requirement A2, inflammation, pneumonia, UCF-101, HtrA2

\section{Introduction}

Pneumonia, a common infectious disease, is characterized by infection of pathogens such as viruses, bacteria and fungi in the lower respiratory tract, and is increasing in incidence and mortality worldwide (1). The typical symptoms of pneumonia are chills, fever, pleuritic chest pain and cough productive of purulent sputum (1). Pneumonia is a major cause of mortality in children $<5$ years worldwide, accompanied with approximately 1.3 million mortalities in children each year $(2,3)$. A better understanding of the mechanism underlying pneumonia in children may aid the development of effective and novel therapeutic approach to overcome this burden.

High-temperature requirement A2 (HtrA2), also known as Omi, is a ubiquitously expressed protein that encodes a serine protein kinase. HtrA2, produced in the endoplasmic reticulum, is located in the inner membrane of the mitochondrial intermembrane space (4). Under apoptotic stimuli, HtrA2 translocates to the cytosol and then promotes cell death by binding to an inhibitor of apoptosis proteins (IAPs), causing caspase activity or promoting caspase-independent cell death $(5,6)$. UCF-101 (5-[5-(2-nitrophenyl) furfuryl iodine]-1, 3-diphenyl-2-thiobarbituric acid) is a specific inhibitor of HtrA2. Previous studies have demonstrated that UCF-101 has a clear protective capacity in organ injury in streptozotocin-induced mouse cardiomyocyte contractile dysfunction (7), dextran sulfate sodium-induced colitis (8) and cecal ligation and puncture-induced septic shock in rats (9). Studies of HtrA2 on pulmonary injury are rare and whether UCF-101 protects lung against apoptotic stimuli remains to be elucidated. Thus, the present study explored the effect of UCF-101 in pneumonia.

The present study identified significant changes of HtrA2 in lung tissues of lipopolysaccharide (LPS)-treated rats. The pathological symptoms and pulmonary injuries were ameliorated by treatment with UCF-101. Investigation of the mechanism suggested that UCF-101 may exert its function by regulating mitochondrial activity and associated gene expression.

\section{Materials and methods}

Animals and experimental design. To induce the pneumonia model, 32 newborn Sprague-Dawley rats (male, 3-8 days old, 8-14 g) were obtained from Zhejiang Center of Laboratory 
Animals and kept in an animal room with prespecified conditions (temperature: $22^{\circ} \mathrm{C}$; relative humidity, $40-50 \%$; $12 \mathrm{~h} \mathrm{light/dark} \mathrm{cycle)} \mathrm{with} \mathrm{free} \mathrm{access} \mathrm{to} \mathrm{water} \mathrm{and} \mathrm{food.}$ The rats were randomly divided into four groups: The control group, without any treatment; the LPS group, which received saline-dissolved LPS $(2 \mathrm{mg} / \mathrm{kg}$; Sigma-Aldrich; Merck KGaA) intratracheally using a miniature nebulizer; the LPS+UCF-101 group, which was injected intraperitoneally with UCF-101 $(2 \mu \mathrm{mol} / \mathrm{kg}$ rats, dissolved in distilled water containing 10\% DMSO) 30 min after LPS administration or the same amount of $10 \%$ DMSO as control for intraperitoneal injection 30 min after LPS administration for the LPS+DMSO group. Then, $24 \mathrm{~h}$ after LPS administration, rats were anesthetized with $30 \mathrm{mg} / \mathrm{kg}$ pentobarbital sodium and abdominal aorta blood was collected. Finally, rats were euthanized using $30 \mathrm{mg} / \mathrm{kg}$ pentobarbital sodium and cervical dislocation. When the rats were verified to have succumbed by lack of heartbeat and cold body, the lung tissue samples were collected for further experiments. All animal experiments were performed in accordance with the animal experimental guidelines set by the National Institutes of Health Guide for the Care and Use of Laboratory Animals (10). The present study was approved by Ethics Committee of Shengjing Hospital of China Medical University (Shenyang, Liaoning, China).

Western blot analysis. The total protein of lung tissue was extracted using RIPA lysis buffer (Invitrogen; Thermo Fisher Scientific, Inc.). The mitochondrial and cytosolic fraction were prepared according to a previous study (11). The concentration of protein was determined using a BCA protein assay kit (Beyotime Institute of Biotechnology). Total protein $(25 \mu \mathrm{g} / \mathrm{lane})$ was separated with $12 \%$ SDS-PAGE and transferred onto a PVDF membrane. The membrane was blocked with 5\% skimmed milk for $1 \mathrm{~h}$ at room temperature, and subsequently incubated with the primary antibodies with a dilution of $1: 1,000$ overnight at $4^{\circ} \mathrm{C}$. The antibodies against HtrA2 (cat. no. ab32092), Bcl-2 (cat. no. ab59348), Bax (cat. no. ab32503), cleaved caspase-3 (cat. no. ab49822), caspase-3 (cat.no. ab13847), cleaved caspase-9 (cat.no. ab2324), caspase-9 (cat. no. ab52298), survivin (cat. no. ab469), X-linked inhibitor of apoptosis protein (XIAP; cat. no. ab2541), cytochrome $c$ (cat. no. ab90529), $\beta$-actin (cat. no. ab8227) and cyclooxygenase (Cox) IV (cat. no. ab33985) were obtained from Abcam. Then, the membranes were incubated with horseradish peroxidase bound to secondary antibody $(1: 5,000$; cat. no. 7074; Cell Signaling Technology, Inc.) at room temperature for $2 \mathrm{~h}$. The protein bands were visualized by an enhanced chemiluminescence (ECL) kit (Bio-Rad Laboratories, Inc.) and quantified using Quantity one 4.6.2 software (Bio-Rad Laboratories, Inc.). $\beta$-actin and Cox-IV were used as the internal controls.

Lung edema. After the rats were sacrificed, the right lung was removed and weighed on an electronic balance to obtain wet weight. After being held at $60^{\circ} \mathrm{C}$ for $48 \mathrm{~h}$, the lung was weighed to obtain the dry weight. The ratio of wet weight/dry weight (W/D) was considered as the degree of lung edema.

Histopathology. The lung tissues were collected and fixed with $4 \%$ paraformaldehyde at room temperature overnight. After dehydrating in graded (70-100\%) ethanol and embedding in paraffin, samples were sectioned at $5 \mu \mathrm{m}$. The sections were then stained using hematoxylin and eosin for $5 \mathrm{~min}$ at room temperature. The histopathological changes were assayed under a light microscope (magnification, $\mathrm{x} 400$ ) from at least three random fields.

Inflammatory cytokine assay. To determine the levels of tumor necrosis factor (TNF)- $\alpha$, interleukin (IL)-6, IL-1 $\beta$ and monocyte chemoattractant protein-1 (MCP-1), ELISA kits (R\&D Systems, Inc.) for IL-6 (cat. no. R6000B), IL-1 $\beta$ (cat. no. RLB00), TNF- $\alpha$ (cat. no. RTA00) and MCP-1 (cat. no. DY3144-05) were obtained and conducted according to the manufacturer's instructions.

Oxidative stress factor assay. To determine the levels of malondialdehyde (MDA), reactive oxide species (ROS), lactate dehydrogenase (LDH) and superoxide dismutase (SOD) the corresponding test kits (Nanjing Jiancheng Bioengineering Institute) were obtained for analysis according to the manufacturer's instructions.

TUNEL assay. Paraffin sections of lung tissue were dewaxed, hydrated, washed with xylene for $5 \mathrm{~min}$ and rehydrated with graded (100-50\%) ethanol. Then, 1\% Triton-100 and 3\% $\mathrm{H}_{2} \mathrm{O}_{2}$-methanol solution was added to the lung tissue sections. After $15 \mathrm{~min}$, the sections were incubated with proteinase $\mathrm{K}$ solution at $37^{\circ} \mathrm{C}$ for $30 \mathrm{~min}$. Subsequently, the lung tissue sections were incubated with TUNEL solution at $37^{\circ} \mathrm{C}$ in the dark for $1 \mathrm{~h}$, then DAPI was added to sections for $10 \mathrm{~min}$ incubation at room temperature. The sections were analyzed with a confocal microscope (magnification, $\mathrm{x} 400$ ) from at least three random fields.

ATP level assay. To determine the mitochondrial adenosine-5'-triphosphate (ATP) level, an ATP-Luciferase Based Bioluminescence Assay kit (Sigma-Aldrich; Merck $\mathrm{KGaA}$ ) was applied to determine the ATP level in accordance with the instructions of the manufacturer.

Statistical analysis. Data are given as mean \pm standard deviation from $\geq 3$ independent experiments. Comparisons among groups were evaluated with one-way analysis of variance followed by the Tukey-Kramer post hoc test. $\mathrm{P}<0.05$ was considered to indicate a statistically significant difference.

\section{Results}

HtrA2 inhibition ameliorates histopathological changes in lung tissues in the LPS-treated rats. To identify whether $\mathrm{HtrA} 2$ was dysregulated in acute pneumonia, the present study utilized a pneumonia rat model induced by LPS to detect the expression level of HtrA2. As shown in Fig. 1A and B, there was an increased expression of HtrA2 in the cytoplasm but a decreased expression of HtrA2 in mitochondria, indicating that $\mathrm{HtrA} 2$ is released from the mitochondria to the cytoplasm in pneumonia. The administration of UCF-101 effectively reversed the dysregulated HtrA2. The histopathological analysis from Fig. 2A showed that the control group had a normal lung tissue structure, and intensive inflammation 
A
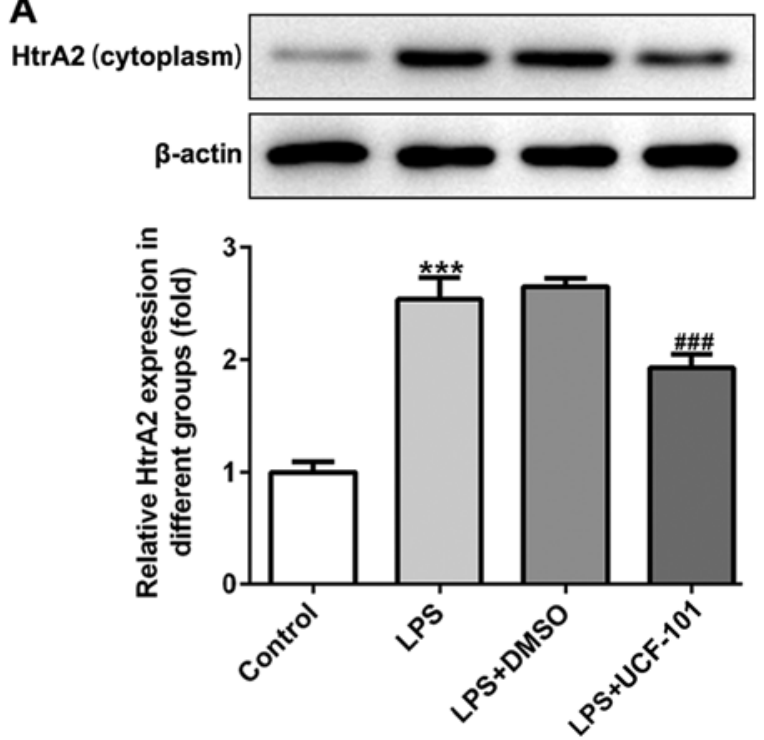

B HtrA2 (mitochondria)
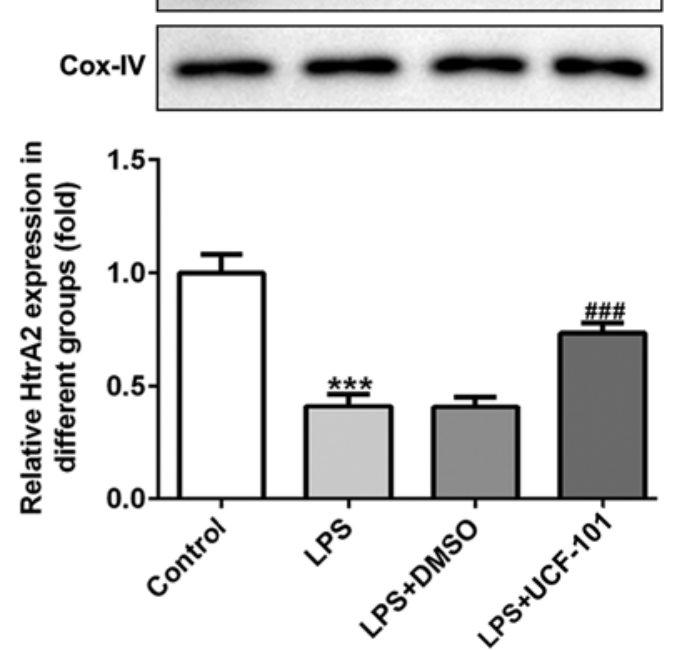

Figure 1. Rats were injected intraperitoneally with $2 \mathrm{mg} / \mathrm{kg}$ LPS to induce a pneumonia model, and were then treated with UCF-101. The protein expression of HtrA2 in (A) cytoplasm and (B) mitochondria was detected using western blotting. Data are expressed as mean \pm standard deviation ( $=8$ ). ${ }^{* * *} \mathrm{P}<0.001$ vs. control, ${ }^{\# \#} \mathrm{P}<0.001$ vs. LPS+DMSO group. LPS, lipopolysaccharide; UCF-101, 5-[5-(2-nitrophenyl)furfuryl iodine]-1,3-diphenyl-2-thiobarbituric acid; HtrA2, high-temperature requirement A2.
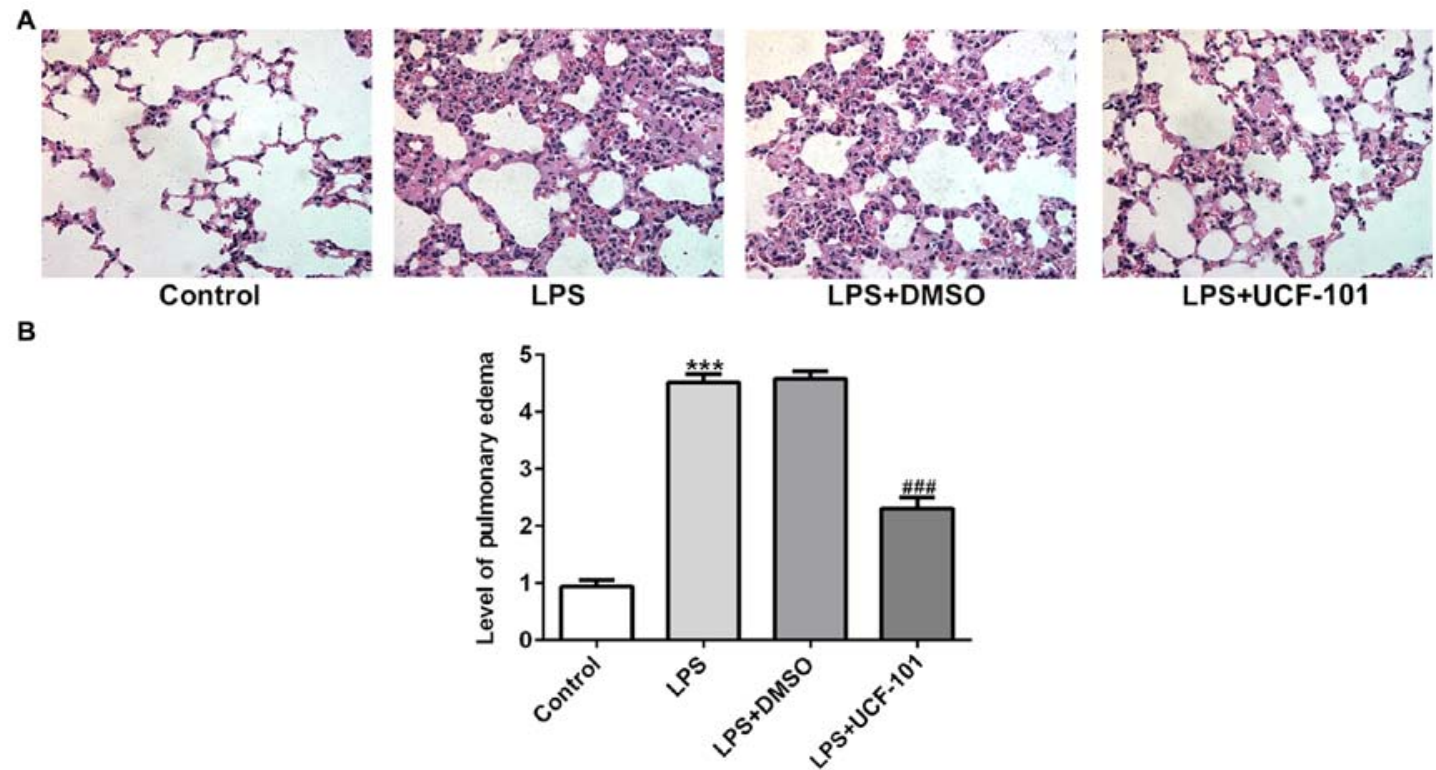

Figure 2. The lung tissues of rats were collected for analysis. (A) The histopathological changes in the lung in the different groups were determined by hematoxylin and eosin staining. Magnification, x400. (B) Pulmonary edema was determined according to the ratio of wet lung/dry lung. Data are expressed as mean \pm standard deviation $(\mathrm{n}=8) .{ }^{* * *} \mathrm{P}<0.001$ vs. control, ${ }^{\# \# *} \mathrm{P}<0.001$ vs. the LPS+DMSO group. LPS, lipopolysaccharide; UCF-101, 5-[5-(2-nitrophenyl)furfuryl iodine]-1,3-diphenyl-2-thiobarbituric acid.

was observed in LPS-induced acute pneumonia rats. The administration of UCF-101 significantly ameliorated these histopathological changes. The level of pulmonary edema calculated by the W/D ratio was significantly increased after LPS stimulation, which was significantly inhibited by treatment of UCF-101 (Fig. 2B).

UCF-101 decreases inflammatory cytokines in the LPS-treated rats. The production of TNF- $\alpha$, IL- $6, \mathrm{IL}-1 \beta$ and MCP-1 were examined to discover the effect of UCF-101 on LPS-induced inflammatory response in pneumonia. In Fig. 3 the levels of TNF- $\alpha$, IL-6, IL-1 $\beta$ and MCP-1 were significantly increased in lung tissues of the pneumonia rat after LPS induction. Treatment with UCF-101 reduced the levels of these LPS-induced cytokines, indicating that UCF-101 alleviated inflammatory response by decreasing inflammatory cytokine expression in LPS-induced pneumonia.

UCF-101 alleviates oxidative stress in LPS-treated rats. The effect of UCF-101 on oxidative stress in lung tissues in LPS-induced pneumonia was also observed. As shown in Fig. 4, the activities of ROS, MDA and LDH 

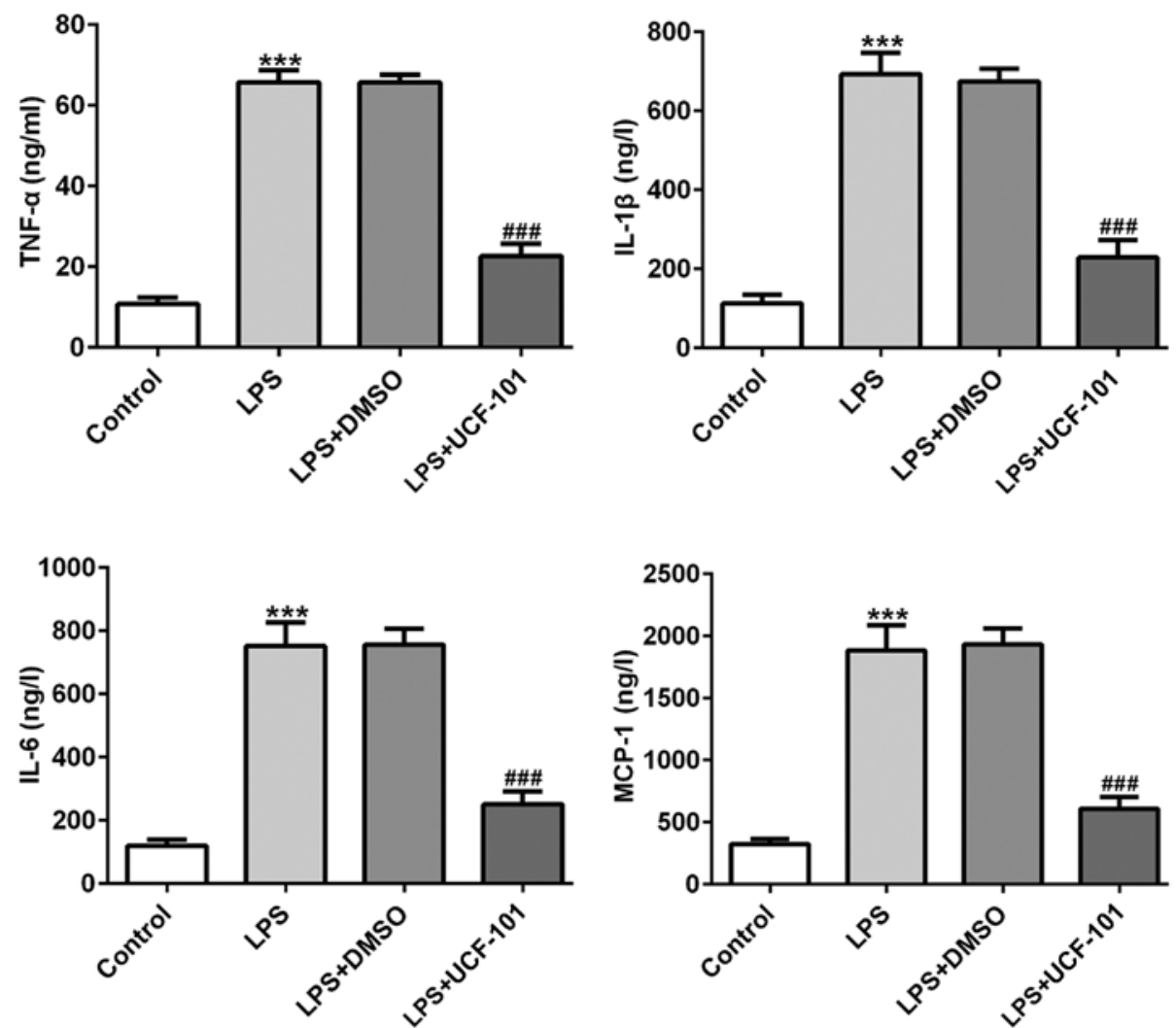

Figure 3. Anti-inflammatory effect of UCF-101 in pneumonia. The levels of TNF- $\alpha$, IL-1 $\beta$, IL-6 and MCP-1 were measured with ELISA. Data are expressed as mean \pm standard deviation $(n=8) .{ }^{* * *} \mathrm{P}<0.001$ vs. control, ${ }^{\# \# \#} \mathrm{P}<0.001$ vs. the LPS+DMSO group. TNF- $\alpha$, tumor necrosis factor $\alpha$; IL, interleukin; MCP-1, monocyte chemo-attractant protein-1; LPS, lipopolysaccharide; UCF-101, 5-[5-(2-nitrophenyl)furfuryl iodine]-1,3-diphenyl-2-thiobarbituric acid.
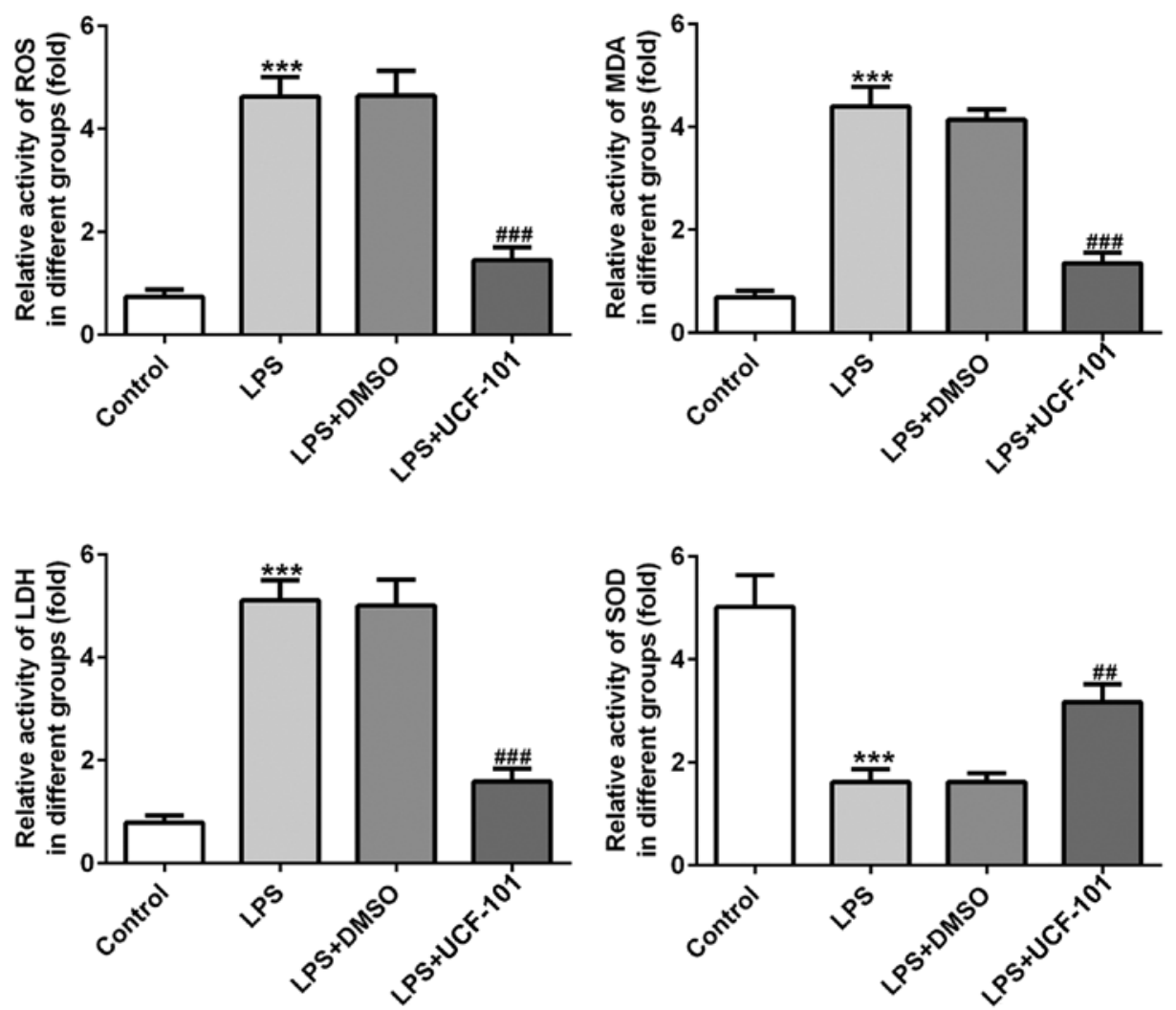

Figure 4. Anti-oxidant effect of UCF-101 in pneumonia. The levels of ROS, MDA, LDH and SOD were measured by corresponding test kits. Data are expressed as mean \pm standard deviation $(n=8) .{ }^{* * *} \mathrm{P}<0.001$ vs. control, ${ }^{\# \#} \mathrm{P}<0.01$ and ${ }^{\# \# \#} \mathrm{P}<0.001$ vs. the LPS+DMSO group. UCF-101, 5-[5-(2-nitrophenyl) furfuryl iodine]-1,3-diphenyl-2-thiobarbituric acid; ROS, reactive oxide species; MDA, malondialdehyde; LDH, lactate dehydrogenase; SOD, superoxide dismutase; LPS, lipopolysaccharide. 


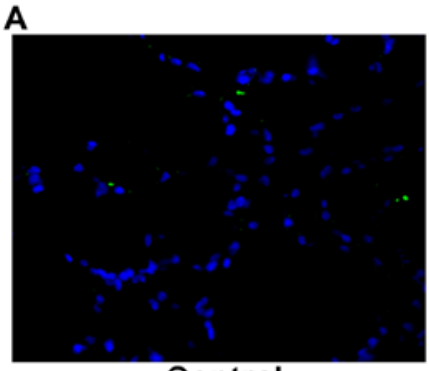

Control

B
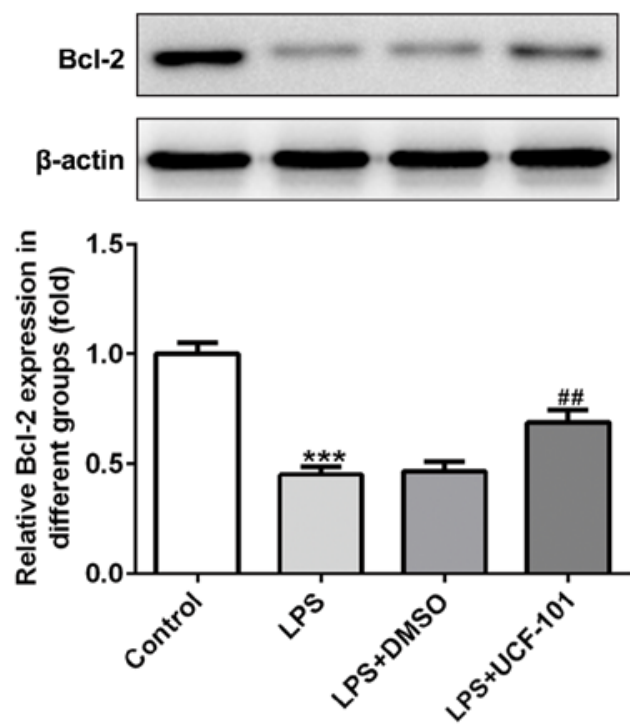

D
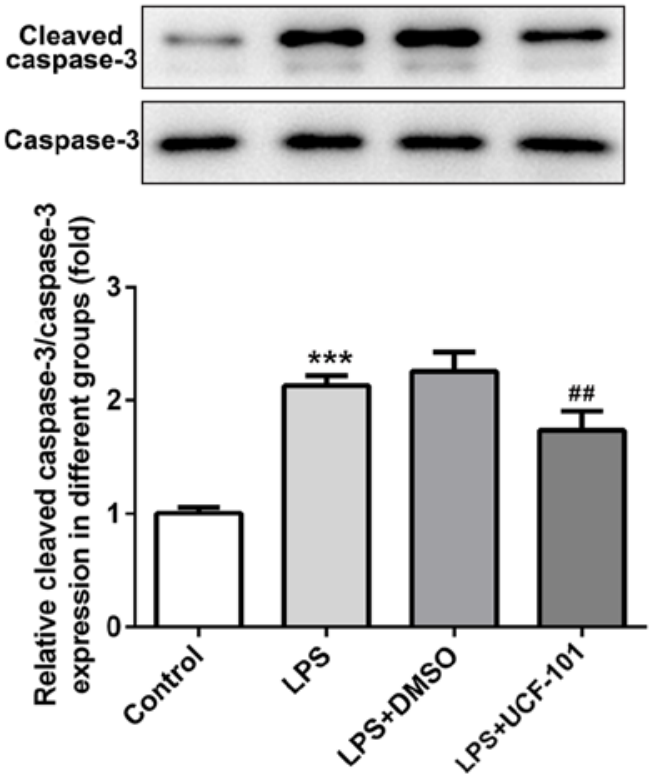

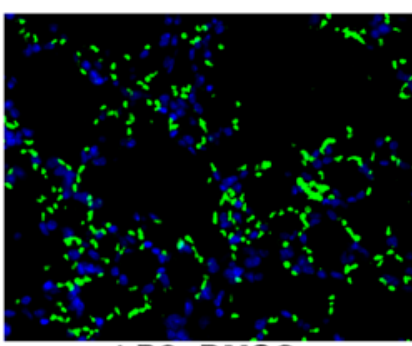

LPS+DMSO

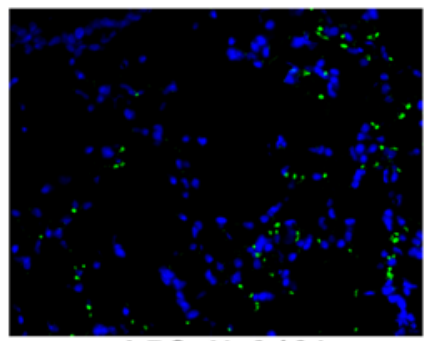

LPS+Ucf-101

C
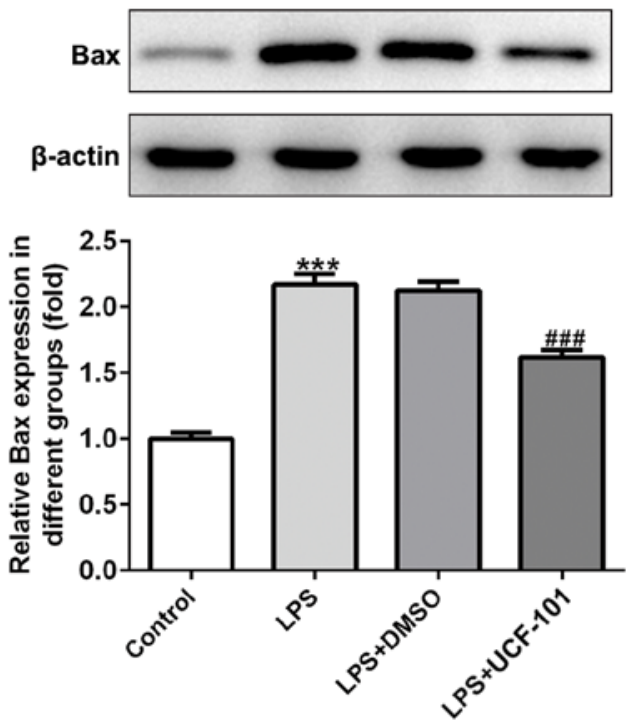

E
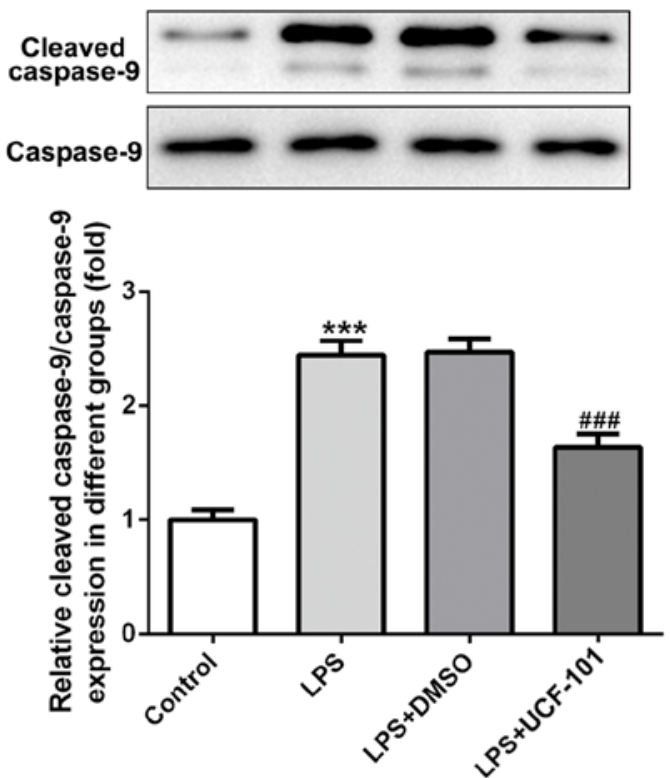

Figure 5. Anti-apoptotic effect of UCF-101 in pneumonia. The condition of apoptosis was detected and (A) the amount of apoptotic cells in lung tissue was determined by TUNEL assay. Magnification, $x 400$. The expression of apoptosis-related proteins (B) Bcl-2, (C) Bax, (D) cleaved caspase-3, and (E) cleaved caspase-9 were determined using western blotting. Data are expressed as mean \pm standard deviation $(\mathrm{n}=8) .{ }^{* * *} \mathrm{P}<0.001$ vs. control, ${ }^{\# \#} \mathrm{P}<0.01$ and ${ }^{\# \# \# P} \mathrm{P}<0.001$ vs. the LPS+DMSO group. UCF-101, 5-[5-(2-nitrophenyl)furfuryl iodine]-1,3-diphenyl-2-thiobarbituric acid; LPS, lipopolysaccharide.

were significantly increased, while the activity of SOD was declined, upon induction of LPS. However, these changes were significantly rescued by the administration of UCF-101. These results indicated that LPS triggered a severe oxidative stress in lung tissue, while UCF-101 was able to alleviate the oxidative stress in the LPS-induced pneumonia rats.
$U C F-101$ reduces cell apoptosis in LPS-treated rats. As apoptosis represents an important phenomenon in pneumonia (12), the apoptosis condition of lung tissue was explored to examine the anti-apoptotic capacity of UCF-101 in pneumonia. A large number of apoptotic cells was found upon the induction of LPS, while the amount of apoptotic cells was decreased by the administration of UCF-101 (Fig. 5A). The expression of 
A
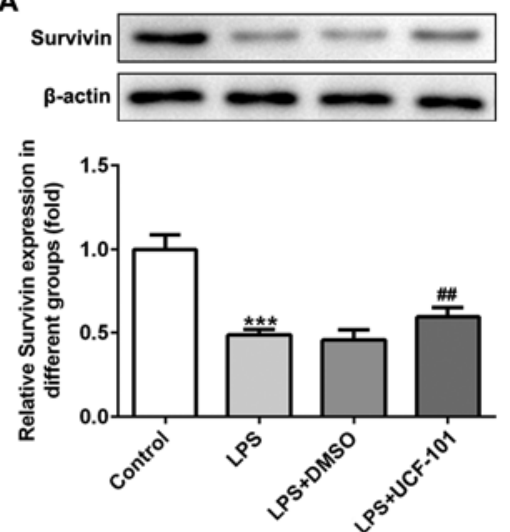

B
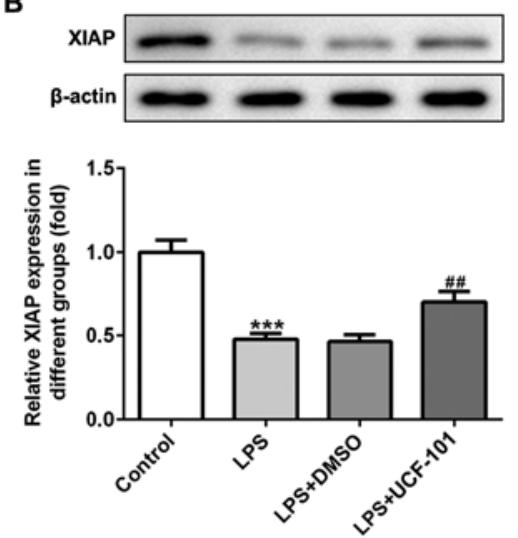
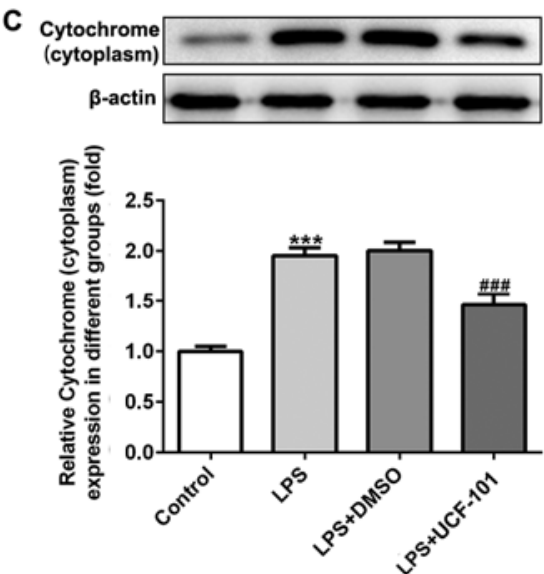

D Cytochrome (mitochondria) Cox-IV

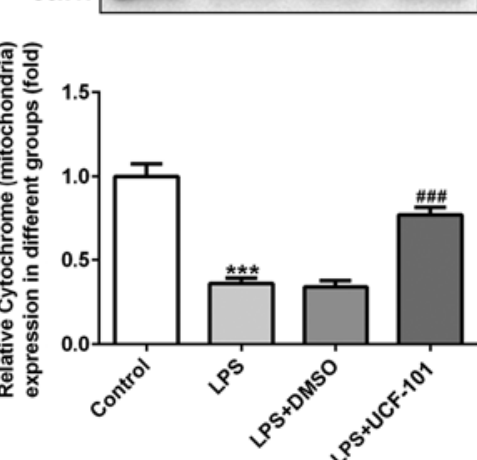

E

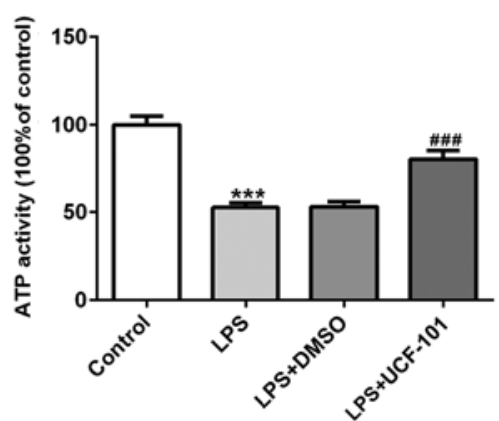

Figure 6. Protective mechanism of UCF-101. The protein expression of (A) survivin, (B) XIAP and cytochrome $c$ in the (C) cytoplasm and (D) mitochondria were determined using western blotting. (E) Mitochondrial ATP level was detected. Data are expressed as mean \pm standard deviation $(\mathrm{n}=8)$. ${ }^{* * *} \mathrm{P}<0.001$ vs. control, ${ }^{\#} \mathrm{P}<0.01$ and ${ }^{\# \#} \mathrm{P}<0.001$ vs. the LPS+DMSO. UCF-101, 5-[5-(2-nitrophenyl)furfuryl iodine]-1,3-diphenyl-2-thiobarbituric acid; XIAP, X-linked inhibitor of apoptosis protein; LPS, lipopolysaccharide; Cox, cyclooxygenase.

apoptosis-related proteins showed that Bcl-2 was decreased, and Bax, cleaved caspase- 3 and cleaved caspase- 9 were increased upon the induction of LPS, which were rescued by the treatment of UCF-101 (Fig. 5B-E), suggesting that marked apoptosis occurred in LPS-induced acute pneumonia, and UCF-101 had a potent anti-apoptotic capacity to alleviate the apoptosis condition.

UCF-101 regulates the mitochondrial apoptosis in pneumonia. The present study next examined the regulatory effect of UCF-101 on mitochondrial apoptosis in LPS-induced pneumonia rats. IAPs can regulate caspase activity, thus affecting apoptosis. Therefore, the expression levels of survivin and XIAP, two IAP family proteins, were detected. As shown in Fig. 6A and B, both survivin and XIAP were downregulated upon the induction of LPS, which was reversed by the administration of UCF-101. The expression of cytochrome $c$ in the cytoplasm and mitochondria was also detected with western blot assay. The results showed that LPS significantly increased the protein expression of cytochrome $c$ in the cytoplasm and decreased cytochrome $c$ in the mitochondria (Fig. 6C and D), suggesting that LPS triggered the release of cytochrome $c$ from mitochondria into cytoplasm. Nevertheless, UCF-101 effectively inhibited the translocation of cytochrome $c$ from the mitochondria into the cytoplasm. Additionally, LPS significantly reduced ATP generation, and the effect caused by LPS was inhibited by the administration of UCF-101 to a certain extent (Fig. 6E).

\section{Discussion}

Pneumonia, a common infectious diseases along with lower respiratory tract infection, is showing increased incidence and mortality all over the world, especially in children (13). Thus, the search for novel and effective therapeutic targets or drugs is urgently required. The aim of the present study was to understand whether UCF-101, a specific inhibitor of high-temperature requirement A2 (HtrA2), has a protective property in lipopolysaccharide (LPS)-induced pneumonia rats. The present study demonstrated that UCF-101 could effectively protect lung tissues against LPS-induced inflammatory response, oxidative stress and cell apoptosis, providing a potential therapeutic drug for acute pneumonia.

Pulmonary inflammation has always been a characteristic of pneumonia (14). Although UCF-101 has been indicated to be anti-inflammatory in the pathogenesis of sepsis complications and colitis $(8,9,15)$, the special anti-inflammatory role of UCF-101 in pneumonia remains to be elucidated. Consistent with the reported studies that UCF-101 suppressed the release of pro-inflammatory cytokines such as TNF- $\alpha$, IL- 6 and IL-1 $\beta$ in colon tissue in colitis, UCF-101 also decreased the levels of TNF- $\alpha$, IL-6, IL-1 $\beta$ and MCP-1 in LPS-induced pneumonia 
rats in the present study. Therefore, UCF-101 appeared to alleviate pulmonary inflammation by inhibiting the production of inflammatory cytokines.

Reactive oxygen species (ROS) play a pivotal role in inflammation (16). Under normal condition, ROS are neutralized by antioxidants so as to maintain the balance of the oxidant and antioxidant system; under pathologic conditions, the level of ROS is expected to be increased and the activity of the antioxidant system decreased, directly leading to an imbalance trending to oxidation, termed oxidative stress $(17,18)$. As oxidative stress is an important process during the pathophysiology of inflammatory diseases, including pulmonary inflammation (19), the present study examined the levels of ROS and antioxidant-oxidant enzymes including MDA, LDH and SOD to verify the effect of UCF-101 on oxidative stress. In agreement with the literature, the induction of LPS significantly induced an elevated level of ROS, MDA, LDH and a decrease of SOD in pneumonia. The administration of UCF-101 effectively alleviated the production of ROS, MDA, LDH and a decrease of SOD.

Cell function and apoptosis are commonly regulated by mitochondria in response to stress stimuli such as inflammatory response and oxidative stress (20). The potential mechanism of how mitochondria function during the process of apoptosis may be important for explaining the protective property of UCF-101 on oxidative stress and inflammatory response in LPS-induced pneumonia. Previous studies have demonstrated that LPS can lead to mitochondrial damage in microglial, blood-brain barrier and even lung tissue, accompanied with injuries, inflammation and cell apoptosis (21-23). In the present study, evident apoptosis occurred in lung tissue upon the induction of LPS, promoting the release of HtrA2 and cytochrome $c$ from mitochondria to the cytoplasm and increasing the expression of pro-apoptotic proteins, which may have been responsible for the subsequent inflammatory response and oxidative stress. The evidence revealed that HtrA2 was able to trigger apoptosis upon its release from mitochondria into the cytoplasm, as well as to trigger the concomitant degradation of XIAP and active caspase- 9 and then downstream caspase-3. Under physiological conditions, caspase- 9 and caspase- 3 are retarded by XIAP to block apoptosis at the post-mitochondrial level (9). UCF-101 was found to suppress the protease activity of HtrA2 to reduce caspase-independent apoptosis (24). Wang et al (15) reported that mitochondrial HtrA2 expression is reduced in murine sepsis, together with a translocation of HtrA 2 from mitochondria to the cytoplasm, while UCF-101 blocks the mobilization of HtrA2 from mitochondria to the cytoplasm, and reduces XIAP, cleaved caspase- 3 and caspase-9 to alleviate sepsis-associated encephalopathy. Hu et al (9) demonstrated the neuroprotective effect of UCF-101 by inhibiting caspase activity and cell apoptosis to attenuate sepsis-induced cognitive dysfunction. As expected, the present study found that cleaved caspase- 9 and cleaved caspase-3 were increased in LPS-induced pneumonia, and this change was reversed by UCF-101. Cytochrome $c$ is considered a caspase activator and is involved in the mitochondrial apoptotic pathway, which is modulated by Bcl-2 family members (25-27). In addition, mitochondria produce ATP for cellular metabolism under a normal condition (28). The reduced ATP level directly modulated mitochondrial dysfunction upon LPS stimulation, which was also reversed by UCF-101. Therefore, the inhibition of HtrA2 was effective in recovering mitochondrial function and restoring mitochondrial-related apoptosis. The administration of UCF-101 had a protective role in LPS-pneumonia by regulating mitochondrial apoptosis.

Overall, the present study indicated that UCF-101 acted as a positive regulator of acute pneumonia by inhibiting the inflammatory response, oxidative stress and mitochondrial apoptosis. The findings suggest UCF-101 as a potential candidate for pneumonia therapy.

\section{Acknowledgements}

Not applicable.

\section{Funding}

No funding was received.

\section{Availability of data and materials}

The datasets used and/or analyzed during the present study are available from the corresponding author on reasonable request.

\section{Author's contributions}

XW designed and performed the experiments, and wrote the manuscript. The author read and approved the final manuscript.

\section{Ethics approval and consent to participate}

The study was approved by the Ethics Committee of Shengjing Hospital of China Medical University.

\section{Patient consent for publication}

Not applicable.

\section{Competing interests}

The author declares that he has no competing interests.

\section{References}

1. Lutfiyya MN, Henley E, Chang LF and Reyburn SW: Diagnosis and treatment of community-acquired pneumonia. Am Fam Physician 73: 442-450, 2006.

2. Agweyu A, Kibore M, Digolo L, Kosgei C, Maina V, Mugane S, Muma S, Wachira J, Waiyego $M$ and Maleche-Obimbo E: Prevalence and correlates of treatment failure among Kenyan children hospitalised with severe community-acquired pneumonia: A prospective study of the clinical effectiveness of WHO pneumonia case management guidelines. Trop Med Int Health 19: 1310-1320, 2014.

3. Bhutta ZA, Das JK, Walker N, Rizvi A, Campbell H, Rudan I and Black RE; Lancet Diarrhoea and Pneumonia Interventions Study Group: Interventions to address deaths from childhood pneumonia and diarrhoea equitably: What works and at what cost? Lancet 381: 1417-1429, 2013.

4. Clausen T, Southan C and Ehrmann M: The HtrA family of proteases: Implications for protein composition and cell fate. Mol Cell 10: 443-455, 2002.

5. Bhuiyan MS and Fukunaga K: Mitochondrial serine protease HtrA2/Omi as a potential therapeutic target. Curr Drug Targets 10: 372-383, 2009. 
6. Ding X, Patel M, Shen D, Herzlich AA, Cao X, Villasmil R, Klupsch K, Tuo J, Downward J and Chan CC: Enhanced HtrA2/Omi expression in oxidative injury to retinal pigment epithelial cells and murine models of neurodegeneration. Invest Ophthalmol Vis Sci 50: 4957-4966, 2009.

7. Li Q, Hueckstaedt LK and Ren J: The protease inhibitor UCF-101 ameliorates streptozotocin-induced mouse cardiomyocyte contractile dysfunction in vitro: Role of AMP-activated protein kinase. Exp Physiol 94: 984-994, 2009.

8. Zhang C, He A, Liu S, He Q, Luo Y, He Z, Chen Y, Tao A and Yan J: Inhibition of HtrA2 alleviated dextran sulfate sodium (DSS)-induced colitis by preventing necroptosis of intestinal epithelial cells. Cell Death Dis 10: 344, 2019.

9. Hu Y, Huang M, Wang P, Xu Q and Zhang B: Ucf-101 protects against cerebral oxidative injury and cognitive impairment in septic rat. Int Immunopharmacol 16: 108-113, 2013.

10. National Research Council (US) Committee for the Update of the Guide for the Care and Use of Laboratory Animals: Guide for the care and use of laboratory animals (8th edition). National Academies Press (US), Washington, DC, 2011

11. Kowluru RA and Abbas SN: Diabetes-induced mitochondrial dysfunction in the retina. Invest Ophthalmol Vis Sci 44: 5327-5334, 2003

12. Qin Z, Yang Y, Wang H, Luo J, Huang X, You J, Wang B and $\mathrm{Li}$ M: Role of autophagy and apoptosis in the postinfluenza bacterial pneumonia. Biomed Res Int 2016: 3801026, 2016.

13. Walker CLF, Rudan I, Liu L, Nair H, Theodoratou E, Bhutta ZA, O'Brien KL, Campbell H and Black RE: Global burden of childhood pneumonia and diarrhea. Lancet 381: 1405-1416, 2013.

14. Wolf L, Sapich S, Honecker A, Jungnickel C, Seiler F, Bischoff M, Wonnenberg B, Herr C, Schneider-Daum N, Lehr CM, et al: IL-17A-mediated expression of epithelial IL-17C promotes inflammation during acute pseudomonas aeruginosa pneumonia. Am J Physiol Lung Cell Mol Physiol 311: L1015-L1022, 2016.

15. Wang $\mathrm{P}, \mathrm{Hu} \mathrm{Y}$, Yao D and Li Y: Omi/HtrA2 regulates a mitochondria-dependent apoptotic pathway in a murine model of septic encephalopathy. Cell Physiol Biochem 49: 2163-2173, 2018.

16. Hussain T, Tan B, Yin Y, Blachier F, Tossou MC and Rahu N: Oxidative stress and inflammation: What polyphenols can do for us? Oxid Med Cell Longev 2016: 7432797, 2016.

17. Floyd RA: Antioxidants, oxidative stress, and degenerative neurological disorders. Proc Soc Exp Biol Med 222: 236-245, 1999.
18. Boskabadi J, Mokhtari-Zaer A, Abareshi A, Khazdair MR, Emami B, Roshan NM, Hosseini M and Boskabady MH: The effect of captopril on lipopolysaccharide-induced lung inflammation. Exp Lung Res 44: 191-200, 2018.

19. Ahmad A, Shameem M and Husain Q: Relation of oxidant-antioxidant imbalance with disease progression in patients with asthma. Ann Thorac Med 7: 226-232, 2012.

20. Tao-Cheng JH: Stimulation-induced structural changes at the nucleus, endoplasmic reticulum and mitochondria of hippocampal neurons. Mol Brain 11: 44, 2018.

21. Zhou D and Jiang Y: Sirtuin 3 attenuates neuroinflammation-induced apoptosis in BV-2 microglia. Aging (Albany NY) 11: 9075-9089, 2019

22. You M, Miao Z, Pan Y and Hu F: Trans-10-hydroxy-2-decenoic acid alleviates LPS-induced blood-brain barrier dysfunction by activating the AMPK/PI3K/AKT pathway. Eur J Pharmacol 865: $172736,2019$.

23. Luo X, Liu R, Zhang Z, Chen Z, He J and Liu Y: Mitochondrial division inhibitor 1 attenuates mitophagy in a rat model of acute lung injury. Biomed Res Int 2019: 2193706, 2019.

24. Cilenti L, Lee Y, Hess S, Srinivasula S, Park KM, Junqueira D, Davis H,Bonventre JV,AlnemriES andZervos AS: Characterization of a novel and specific inhibitor for the pro-apoptotic protease Omi/HtrA2. J Biol Chem 278: 11489-11494, 2003.

25. Saelens X, Festjens N, Walle LV, van Gurp M, van Loo G and Vandenabeele P: Toxic proteins released from mitochondria in cell death. Oncogene 23: 2861-2874, 2004.

26. Brunelle JK and Letai A: Control of mitochondrial apoptosis by the Bcl-2 family. J Cell Sci 122: 437-441, 2009.

27. Lin PY, Tsai CT, Chuang WL, Chao YH, Pan IH, Chen YK, Lin CC and Wang BY: Chlorella sorokiniana induces mitochondrial-mediated apoptosis in human non-small cell lung cancer cells and inhibits xenograft tumor growth in vivo. BMC Complement Altern Med 17: 88, 2017.

28. Krause J, Löser A, Lemoine MD, Christ T, Scherschel K, Meyer C, Blankenberg S, Zeller T, Eschenhagen T and Stenzig J: Rat atrial engineered heart tissue: A new in vitro model to study atrial biology. Basic Res Cardiol 113: 41, 2018.

(i) $(2)$ This work is licensed under a Creative Commons Attribution-NonCommercial-NoDerivatives 4.0 International (CC BY-NC-ND 4.0) License. 